\title{
Accessibility approach to Web applications development: An Experimental Study
}

\author{
Thiago Jabur Bittar ${ }^{1}$, Luanna Lopes Lobato ${ }^{1}$, Renata Pontin de Mattos Fortes ${ }^{2}$ \\ ${ }^{1}$ Computer Science Department, Federal University of Goiás - Catalão, GO - Brazil \\ ${ }^{2}$ São Paulo University - ICMC - São Carlos, SP, Brazil \\ \{jabur, 111$\}$ @catalao.ufg.br, renata@icmc.usp.br
}

\begin{abstract}
Despite the number of studies available in the literature, about the use of accessibility during Web application development, there are some gaps related to lack of empirical results about it, and so, accessibility barriers are intrinsically present in the organizational culture. In order to contribute to the accessibility domain, we developed an approach used to provide support and facilitate the use of accessibility during Web applications development. In this paper, we presented the results identified through the use of such approach in a controlled experiment. In this sense, we could verify the benefits and drawbacks with the use of it, and so propose improvements for it. This experiment was carried out with the contribution of participants, which were divided in two groups. We understand that such scenario may not enable us to generalize our findings to a diversity of scenarios, however, this study could serve as an initial point towards validating our approach, and may serve to establish baseline values for future studies.
\end{abstract}

\section{Introduction}

The lack of accessibility has become a prevalent issue that challenges developers of web applications. Through the implementation of good accessibility practices it is possible to remove the barriers that prevent certain groups of people from accessing information. In other words, accessibility is an attribute which ensures that an application is usable by everybody, even by people with disabilities. For this reason, accessibility has become an essential requirement for web applications [1].

Accessibility is very important to software applications and several studies have been dedicated to it. In spite of this, the current scenario presents a mismatch between the requirements imposed to apply accessibility during system development and what is really performed and presented to the market. Thus, there is a gap related to accessibility in web applications development due to the lack of follow the guidelines and recommendations which, once adopted, would provide access for many users [1][8].

In this way, assistive technologies may help people to interact with a software application, but only if the application is developed in compliance with accessibility standards. It is relevant to mention that the maturity of the user should be considered, since it is crucial to the satisfactory use of the applications. Thus, assistive technologies mediate the interaction between the user and the application. However, even when using 
such technologies, many users are restricted from accessing information and performing online services due to accessibility barriers.

For this reason, our study is focused on the use of good accessibility practices, since there is an increased awareness about the topic and a growing demand for applications that take into consideration the needs of all potential users. To ensure the development of accessible web applications, it is important to train developers. In view of this, this study proposes and presents an approach to guide developers during system's development. In this way, they will learn what must be done in several cases to ensure accessibility to web content.

In order to test the proposed approach (specific to web development), a controlled experiment was undertaken in an academic environment, involving undergraduate students from the Computer Science Department of Federal University of Goiás. First of all, the students were trained and after they were divided in groups to perform a task. A questionnaire had been previously applied to identify the participants' profiles so that they could be distributed in similar groups. The groups were asked to develop a web application for scheduling scientific evaluation committees. The experiment resulted in several considerations and useful findings that contributed to improve the approach. Such results were shared among the development teams. As a result of this study we identified an increasing awareness of the participants relating to accessibility issues. This is relevant since the academy and the industry can take profit of the insights presented in this study and incorporate the lessons learned to new web projects.

The remainder of this paper is organized as follows: Section 2 presents the related work. Section 3 presents the concepts about accessibility and Design Rationale. Section 4 summarizes the approach developed and assessed in the experiment presented in this paper, and Section 5 describes the methodology used. Section 6 present experiment conduction and results, followed by the summary of findings and conclusions, presented in Section 7 and 8, respectively.

\section{Related Work}

The need of improving collaboration in design decisions, refining the requirements traceability and optimizing the use of new technologies is discussed in [7], which reports the development of a technique to boost documentation goals and to register the motivations for decisions. This research is used as base of our study, since it can contribute in order to present ways that can be followed to document decisions collection through experimental evaluation.

Gunderson et al. present accessibility assessment tools and detail what is necessary to achieve the expected results. Additionally, in [2] the authors define some assisting guidelines developed to ensure efficiency and portability during the websites development. However, they are not specific to tackle accessibility issues on web software development.

For the best of our knowledge, an experiment using an approach to support the use of accessibility during Web application development is not known. Thus, we looked for different domains and studies assessment approach in controlled experiment. 


\section{Web Accessibility and Design Rationale: Concepts and Guidelines}

Accessibility can be interpreted as the possibility of using a resource universally, without barriers or through alternative access means. In the web context, the resource to be accessed is constituted by the pages content. Thus, accessibility refers to the inclusive practice of making websites available for people of all abilities and disabilities. The idea of this concept is that the users, using every agent, can understand and interact with the offered content [16].

Currently, there is a significant gap of knowledge from developers and experts in accessibility. Most programmers have no necessary knowledge or experience to ensure that their code attends the accessibility requirements [8]. Particularly, in web projects, it is a common practice to consider accessibility only at the advanced stages of development or when the applications are entirely coded. At this point, making applications accessible is a real challenge that involves redesign and reprogramming [13]. Moreover, developers almost always do not know details about accessibility practices or, if they do, not use such knowledge during the application development. Thus, most programmers have no essential knowledge or experience to ensure that their code meets accessibility requirements.

A solution for this problem is the use of lessons learned from other projects, since the same mistakes may be avoided and the accessibility practices already tried with success may be reused. This solution reduces costs related to the process of choosing the practices that should be applied during the application's development. For this reason, in general, the use of patterns and templates is recommended.

However, according to [1] very few developers have been explicitly trained for accessible pages creation. Evaluating pages using assistive technology may reveal problems related to the complexity of defining systems and thus verify the real benefits of its use.

Aiming to explain how to produce accessible web contents, the World Wide Web Consortium (W3C), through its Web Accessibility Initiative (WAI), released a collection of accessibility guidelines in a document named Web Content Accessibility Guidelines (WCAG). These guidelines present recommendations to produce web content (texts, images, forms, sounds) accessible for people with disabilities including blindness and low vision, deafness and poor hearing, learning difficulties, dyslexia, cognitive limitations, movement limitations, speaking inability, photosensitivity and combinations of them. The WCAG 2.0 presents four principles that form the foundation of Web accessibility: perceivable, operable, understandable and robust. From these principles, 12 recommendations are presented in $\mathrm{W} 3 \mathrm{C}$ [16]. The 12 recommendations are divided into three levels of conformance: A (minimum), AA (medium level) and AAA (highest level).

As a complementary support, the Design Rationale (DR) framework can be defined as a reference for the reasoning that justifies a project as well as for discussions that justify the choice of structure on other alternatives [9][12][14]. In [12][11] is presented that DR not only includes a description of the potential artifact, but also defines reasons for decisions, experiences, alternatives and arguments that lead to the decisions that best fit to the system development. 
The DR framework may be used to capture and manage architectural knowledge, which is extremely important in software projects, since it includes information from the environment and reasons for the process of design and negotiation, defining the outcome as the final product [18]. However, storing DR can take a significant time and be expensive, since the most current DR system can fail to consider practical concerns, such as cost-effective use and smooth integration [11]. In order to solve these problems, it is possible to automate the capture process and associate it with the work object, i.e. the development itself. The use of DR encourages the learning of a project as a whole and may represent a solution to help designers to identify issues that might otherwise go unnoticed [9]. It also contributes to the identification of inappropriate premises besides reducing the designers' tendency of ignoring possible alternatives for important decisions.

Knowledge is recognized as a decisive factor to develop accessible software applications. For this reason, it became the focus of institutions that need to use this kind of application, making decisions constantly. Likewise, the DR is recovered to capture and record decisions made on projects and their respective impacts, good or bad. This occurs because such decisions can lead to success or failure in a project. Documenting experiences is important to build a knowledge base for future projects through lessons learned, in order to avoid rework and repetition of the same mistakes, enabling better decisions [2][6].

Other useful employment of this methodology, according to [6], is promoting collaboration and knowledge sharing among team members, facilitating maintenance and reuse, improving the quality of the artifacts and supporting new design decisions.

The DR concept was selected to assist the use and register of decisions and techniques concerning web accessibility, since we aim to build a useful basis for knowledge management and reuse by the work team.

\section{The Approach}

In this section we present an overview of the approach developed to support the use of the accessibility guidelines during Web applications development. The approach was proposed to solve the problems identified by the lack of consideration of issues related to Web accessibility.

The approach is based on the studied theoretical principles (related to the concepts involved) and results from practical studies (related to empirical studies) performed along the research. Thus, it was based on findings identified through studies on Accessibility and Web Engineering areas. The approach has been formulated since 2010 [2][3][4], and it has been developed following a top-down methodology, incrementally, based on our previous findings and refined according to improvements were identified.

Using the approach it is possible to plan and implement the actions to correct the possible problems that can occur, mainly regarding to the accessibility barriers. Some actions were proposed to define the necessary efforts related to:

- Educate the development team making the learning related to how apply the best accessibility practices possible; 
- Provide training, aimed at disseminating information among the team in order to facilitate the accessibility practices adoption;

- Manage decisions related to development with accessibility, reuse successful solutions through consulting and training from a base of historical facts;

- Elicit accessibility requirements and document what is being developed in the project, according to the users profiles and business needs identified;

- Choose suitable tools to develop the project and use its resources in a effectively way, in order to facilitate and optimize the accessibility, and

- Reuse software components already developed and with accessibility verified.

The approach is oriented in three layers, which were defined to group similar activities related to actions that must be followed to apply appropriated accessibility practices. These layers are: 1 - AT. Accessibility Training, 2 - DM. Decisions Management, and 3 - DT. Development and Tools.

The first layer (AT. Accessibility Training) was defined due to the need in educate the stakeholders involved in the development of Web apps regarding to the accessibility issues. This layer was based on the work presented by [8], where the authors had verified that a considerable number of practitioners did not know about the accessibility guidelines, and, therefore, did not make use of them.

Regarding the training layer, as suggested by the approach, it is necessary to provide access to the reference materials, such as recommendations and accessibility guidelines. Due to extensive quantity of material available in the Internet and the lack of pattern relate to the quality of them, it is necessary a previous selection to define which materials are appropriate and could be useful as a support to the learning. This selection should not exclude the search for additional material by the developers; however, it is essential to verify the reliability and adherence of each material according to the practices and way adopted by the organization to develop software. Thus, the material already validated should be prioritized to be used in the project.

A relevant point is that examples, about Web development with accessibility, should be presented to the professionals that will develop the project. The examples should be clear and consistent, showing common accessibility problems and their consequences in the practice, regarding the advantages and disadvantages for the project.

The second layer (DM. Decisions Management) is justified due to the importance in taking consistent decisions related to the development process with accessibility best practices. It is observed that there are different methods and strategies to adopt such practices. However, wrong choices could jeopardize the project progress. Thus, it is expected that quality and control criteria should be considered into the application, in this layer, related to the necessary decisions about the evaluations discussed on accessibility issues by the developers.

One of the main challenges in this area is to make good decisions, with the appropriate foundations, on which results can be achieved with the execution of specific actions. The foundations may be obtained by consulting the historical and 
methodological factors. Regarding to the historical verification, the reuse of solutions that have reached success in the past is appropriate, since they were achieved through lessons learned from other projects. In fact, it helps to predict consequences and to reuse a successful work already performed.

Finally, the third layer 3 (DT. Development and Tools) was defined because defining which actions will be implemented is a necessary task as well as to put them into practice during web apps development. Thus, it is necessary to take into account the support of assistive tools, which present important resources to make the development in an agile and reusable way possible. In addition, in the third layer, it is suggested the use of facilitators with concerns about accessibility in the development environment. This is important to the aware developers using the resources effectively in authoring and evaluation tools. These facilitators can be the usage of validators, alerts during the development time, search of design patterns and components use.

Another important issue in this approach is related to the reuse of components, in order to improve the accessibility. Implementations patterns, already developed by the company or partners, can be used as components, since the accessibility is guaranteed. This can be verified through test to each component.

The inclusion of information about good practices in the models and transformers enables the generation of code with the solutions applied in an efficient and a semi-automatic way.

\section{Methodology}

This section presents a controlled experiment performed with the aim of assessing the benefits and drawbacks of the reported approach. The experiment was carefully designed and validated by two main researchers: (i) a Ph.D. expert in empirical Software Engineering, who helped to define the processes to be followed when developing and running the experiment; and (ii) a Ph.D. student that has worked with web development and accessibility in the last five years, who is the main author of this paper and who proposed the approach.

In this study we developed an academic experiment since experiments in a real scenario demand several external interventions, take more time to be performed and require additional investments. Moreover, it is difficult, sometimes, to find companies that agree to undertake academic experiments in their development process.

The methodology used in this study consists in executing a sequence of stages, like the general planning, the analysis of developers experience and their division into groups aiming to have a technical balance on each team. To define the stages and to set other methodological details, we used the recommendations, concepts and techniques for experiments described in [10].

In order to perform a systematic experiment, we followed the recommendations defined in Wohlin et al. [19], which describe the plan, or protocol, used to perform the experiment and also to analyze its results. The planning phase consists of seven steps (context selection, hypothesis formulation, variables selection, selection of subjects, experiment design, instrumentation, and validity evaluation). 
Figure 1 presents the scheme to perform the experiment, where are showed the steps followed, as the identification of the subjects' expertise, the definition of the groups, as well as, other activities related to the experiment development and the assessment of the identified results.

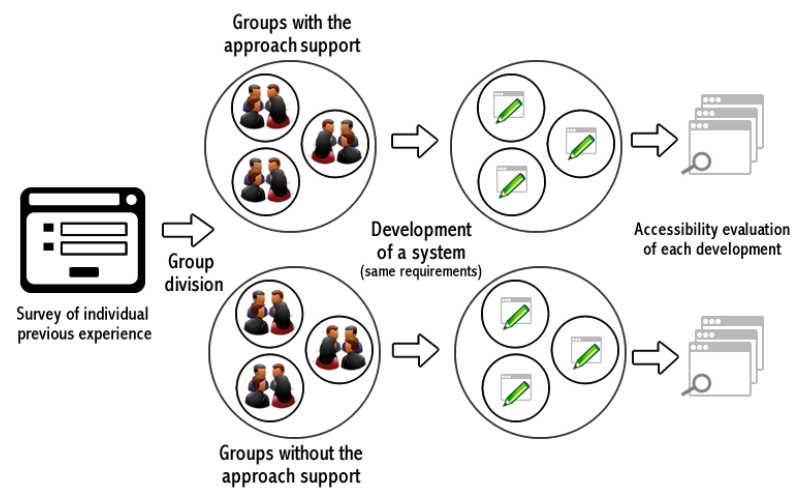

Figure 1. Scheme to perform the experiment

As presented in Figure 1, after the groups definition, the development of a system was required to the groups, with the same requirements imposed as mandatory to each one. This scenario was required since the goal was to contrast the findings presented by the two groups, to assess the effect caused by the usage of the proposed approach. Thus, it was possible to map the results, in order to verify the benefits and drawbacks with the approach support to apply accessibility. The next subsections present in details the methodology used and the steps followed.

\subsection{Instrumentation}

In order to guarantee that the experiment was well calibrated, two pilot projects were conducted with the same structure defined in the planning before performing the experiment with the subjects. The first pilot aimed to detect possible problems in the experiment and calibrate it before its execution. Some issues were verified, as those regarding how to apply the accessibility's guidelines during the development of web applications. It depends on the developers' perception and the context of the project to which the approach is applied.

The second pilot was performed by a single subject, who has experience in developing experiments in Software Engineering and has sufficient knowledge about accessibility, web development, and empirical studies. We detected some problems related to the artifacts developed as support to this experiment and implemented some modifications in order to solve them. In addition, we also identified problems with the forms (used to assess the expertise of the participants). Based on the results of this second pilot study, we added some definitions to evaluate the approach and removed or modified some considerations.

\subsection{Selection of subjects}

The subjects of this experiment were selected by convenience sampling, which means that the nearest and most convenient persons were selected as subjects to participate in 
the study [19]. We performed a controlled experiment in the academic context, selecting graduate students as participants, which acted as developers.

Initially, all subjects attended a training session on accessibility, which included the study of the guidelines and the discussion and evaluation of tools. This training involved the presentation of the WCAG guidelines, used to develop more accessible applications, as well as oral discussion on related papers.

\subsection{Operation}

In order to guide the task, the requirements and the relational database proposed for the desired application were presented to the participants. The database is compound by seven tables, intended to be an organized repository for the data required by the application. Such application should allow the schedule of committees' assessment sessions of undergraduate final projects. In summary, the entities (with their attributes) of this application are:

- Committees: which have a chairman and other members (usually two), a date, a room, a presenter and a work data to be presented;

- Rooms: which have a name, a code and a location;

- Persons: which have a name, an e-mail, a phone number, an institution and a password.

The following business rules were established:

- It is not allowed the presence of two committees at the same time and place;

- Any person is allowed to be at the same time the committee chairman and a member.

The tables, defined in the application database, present the relationship between them: "room", which makes reference to the place where the presentation happens; "person", which may be the presenter, a participant or the administrator (these roles are defined by the relationship of the "profile" and "person_profile" tables); "evaluation_committee": which has the presentation start time, end time and the final score. Finally, there are two other relationship tables: "committee_person" and "function_committee", associating evaluators and defining their specific roles (chairman or member).

All groups were instructed to perform the DR capture, record decisions and important information from the development and post them in a Wiki page, using the following format: i) topic; ii) creation date and author name; iii) motivation for the topic creation; iv) comments from each member of the team and, finally, v) the team final decision. The Wiki was used to report the results and make available the lessons learned for future projects.

In the sequence, to enable comparison, we separated the teams that would use the approach and the teams that would not use it. The teams, drawn by lot, to use the approach were the Groups 2, 3 and 5.

Regarding requirements, the desired application was expected to be accessible, interactive and usable, with rich elements such as dropdown menus and an interactive 
calendar to help choosing the date. We set a deadline of two weeks for artifacts delivery. This period is considered short and it was set to simulate the conditions usually found in software development companies' environment.

For the final evaluation, a set of criteria were defined to assess the artifacts produced by each team. These criteria follow a set of WCAG 2.0 recommendations, as presented in Table 1. The codes in brackets are related to the section on specific techniques to accomplish certain success criteria [17].

Table 1. Established Accessibility Criteria

\begin{tabular}{|c|c|}
\hline Established criteria & Relationship with WCAG 2.0 \\
\hline 1 - Accessible tables & $1.3 .1(\mathrm{H} 73, \mathrm{H} 39$ and H51) \\
\hline 2 - Images with alternative text & $1.1 .1(\mathrm{H} 37$ and H67) \\
\hline 3 - Forms with appropriate labels & $1.1 .1,1.3 .1,3.3 .2$ and $4.1 .2(\mathrm{H} 44)$ \\
\hline $4-$ Correct use of header tags (h1, h2, ..) & 1.3 .1 and 2.4.10 (G141 and H52) \\
\hline 5 - Elements with good contrast & 2.4 .3 \\
\hline 6- Proper use of title on pages & 3.2 .3 \\
\hline 7 - Absence of broken links & \\
\hline
\end{tabular}

As may be seen in Table 1, we chose a subset of the WCAG 2.0 criteria for evaluation, aiming at facilitating the task and eliminating several items that were not applicable to our domain, as video and audio guidelines, for example.

\section{Experiment Conduction and Results}

The controlled experiment had 24 participants and they were asked to answer a questionnaire about their experience in accessibility, development and software engineering.

Based on the answers, we analyzed their expertise and divided them into six balanced groups of four members. We tried to keep the groups with the same level of technical expertise.

In order to measure the benefits of the approach developed, some groups used it and other groups did not. Two scenarios were defined in this experiment. In the first one, the use of the approach was considered, presenting to the subjects some artifacts that could be used to facilitate the development. In the second scenario the approach was not used as support to the experiment, and so the artifacts of the approach were not provided to the subjects. Thus, the design used in the experiment is one factor with two treatments, and these two treatments are compared against each other [19].

The experiment was conducted in an undergraduate laboratory during 64 hours, between January and February 2012, when accessibility concepts and their guidelines were presented to the participants. After this, the participants performed the code development.

All groups succeeded in developing the application, but they did it at different levels of completeness and met different accessibility requirements. Detailed explanations of each group's resulting application are made in the following:

- Group 1: We found serious errors in the artifacts developed by Group 1. These errors include incorrect spelling, images without alternative text to help and 
forms with problems of accessibility. Figure 2 presents a sample of the design performed by this group. Through these findings it is possible to conclude that the use of guidelines to accessibility is relevant to optimize the development of accessible applications, reducing time and cost during the development.

- Group 2: Since the Group 2 was selected to use the approach, we expected to found more satisfactory results than in Group 1. This was confirmed once a correct use of heading elements $\mathrm{H} 1$ and $\mathrm{H} 2$ was made. In its development, as presented in Figure 3, there are other positive items that we can highlight, as: A drop-down menu accessible via keyboard; Elements to control the text size and contrast (as seen at the top of the figure); Form labels correctly done; and Images with alternative text to help during the filling of the form. The findings identified in the artifact produced by Group 2 confirmed the supposition that using guidelines and good practices of accessibility contributes to accessible software applications development.

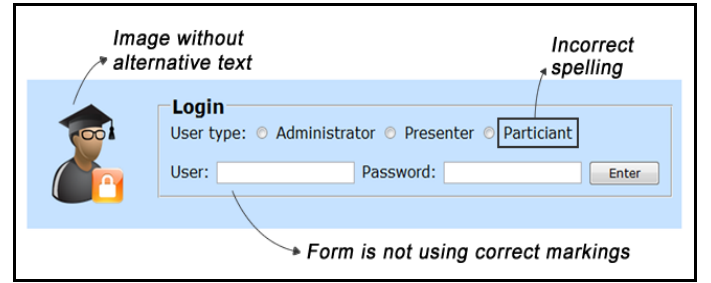

Figure 2. Design with problems at login page of Group 1

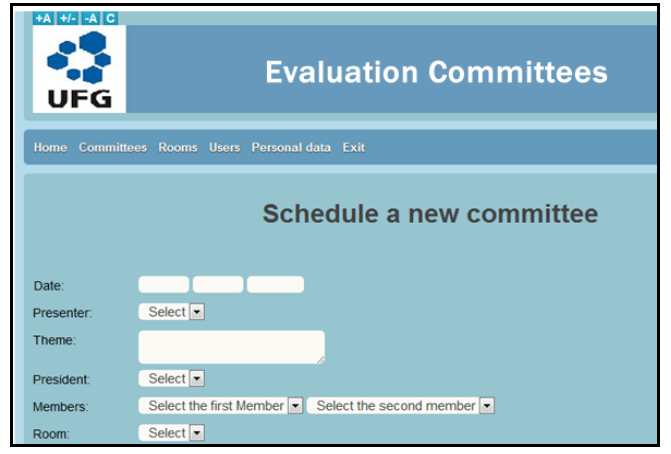

Figure 3. Group 2 accessible design

- Group 3: It made use of H1 tags for titles in a correct way, and pointed the mandatory fields to be filled in the forms. A sample of this design is shown in Figure 4, where $\mathrm{H} 1$ is to titles, however there is a problem related to the contrast in the header. As we can observe, there is a problem in the header, with the color contrast. A contrast ratio 2:4:1 was found, failing to fulfill WCAG guidelines in accordance to levels AA and AAA (success criterion 1.4.3). Another issue to be highlighted is the existence of tables with accessibility problems, where there is no marking of headers or summary.

- Group 4: Concerning Group 4's artifacts, their tables were correctly made using the tags "summary" and "header". However, the implementation had a very simple interface and this might not satisfy the user in what refers usability. This development is presented in the Figure 5, where is presented the use of accessibility guidelines for the table design (the source code is shown, but poor layout is verified). The forms are properly made using labels and there is a validation resource in their fields. These validation resources are efficient since they show the mistakes that the user can make, optimizing the filling of the form and contributing for the finalization of the task. 


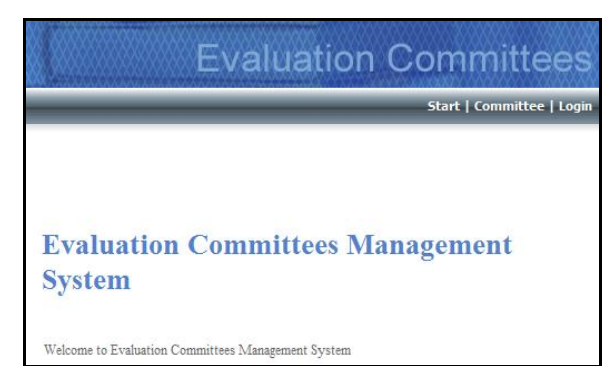

Figure 4. Problem with contrast problem in the header

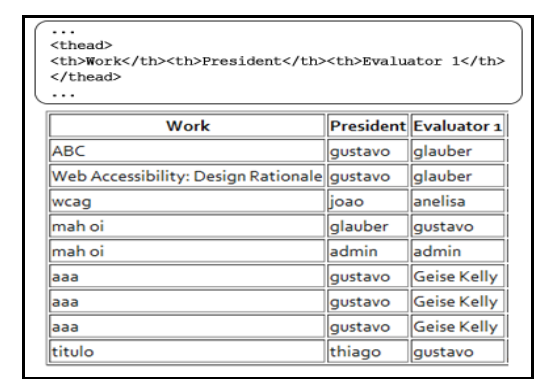

Figure 5. Use of accessibility guidelines for the table design

- Group 5: In this group's artifacts we found a drop-down menu, accessible via keyboard, and tables properly constructed. The forms were correctly made with labels, mandatory fields marking and JavaScript client-side validation. Regarding the negative aspects, some language mistakes and poor layout must be mentioned. There are two possible explanations for these shortcomings: the participants might not have paid attention to the task of filling the fields or they lack some skills due to educational deficiencies since they came from different schools. However, despite this problem, this group presented the best evaluation according to the checked criteria. We believe that the approach used was the main reason for this result.

- Group 6: In the artifacts produced by the Group 6 it is possible to verify the correct use of $\mathrm{H} 1$ title tag. However, the use of the listing data has a very small font size and the tables were not made accessible. The Group 6 suggested changes in the database, aiming to simplify it, as presented in the following four tables: "committee", "person", "room" and "occupied_room". However, this intervention reduced the system flexibility and changed the initially established requirements. The motivation for this suggestion was reported in the DR, as follows: "We made changes in the database to facilitate our life; we merged some tables and created others. This action has been taken to speed up the system construction, since we were short of time". This documentation is significantly important since it makes the performed changes and their respective reasons available for the rest of the development team. As a positive element, we found properly made labels. However, some images do not have alternative text.

\section{Summary of Findings}

In order to present the results of this study, we related the findings identified and the groups that reported them. Table 2 summarizes the assessment of the artifacts developed by each one of the groups, where A means "Use of accessible tables"; B - "Use of images with alternative text"; C - "Forms with appropriate labels"; D - "Correct use of header tags"; E - "Elements with good contrast"; F - "Proper use of title on pages"; G "There are not broken links".

It is possible to observe in Table 2 that no criterion was fulfilled by all the groups. To verify the quality of the insights from each group we performed a quantitative evaluation. This evaluation was based on the criteria defined in this paper. A value 1 was assigned for each fulfilled criterion and a value 0 was assigned when the development was not in accordance with the criterion. In order to obtain the score of 
each group $\mathrm{j}$ (called as $\mathrm{SGj}$ ) was calculated the metric to the associated values, which is expressed by the Equation 1.

Table 2. Established Accessibility Criteria

\begin{tabular}{|c|c|c|c|c|c|c|c|}
\hline & \multicolumn{7}{|c|}{ Criteria (i) } \\
\hline Groups (j) & A & B & C & D & E & F & G \\
\hline $\mathbf{1}$ & -- & -- & -- & -- & $\checkmark$ & -- & $\checkmark$ \\
\hline 2 & $\checkmark$ & $\checkmark$ & $\checkmark$ & $\checkmark$ & $\checkmark$ & $\checkmark$ & -- \\
\hline $\mathbf{3}$ & -- & $\checkmark$ & $\checkmark$ & $\checkmark$ & -- & $\checkmark$ & -- \\
\hline $\mathbf{4}$ & $\checkmark$ & -- & $\checkmark$ & $\checkmark$ & $\checkmark$ & -- & -- \\
\hline $\mathbf{5}$ & $\checkmark$ & $\checkmark$ & $\checkmark$ & $\checkmark$ & $\checkmark$ & $\checkmark$ & $\checkmark$ \\
\hline $\mathbf{6}$ & -- & -- & -- & $\checkmark$ & $\checkmark$ & -- & - \\
\hline
\end{tabular}

$$
\mathrm{SG} j=\sum_{i=1}^{7} \mathrm{X} i j
$$

Equation 1. Calculate

the scores

According to Equation 1, Xij is the value to be calculated, where i represents the index to the criteria (related to the lines) and $\mathrm{j}$ represents the group (related to the columns). To achieve the group score, the index $\mathrm{j}$ is fixed and are counted the values associated to each criterion, starting from $i=1$ (A) to $i=7(G)$. The final scores are presented in the Figure 6, where demonstrates the groups using the approach presented higher scores than those not using the approach. However, Groups 3 and Group 4 obtained the same score in spite of the first one having used the approach and the second having not used it. This is justified because using the approach constitutes an aid, not a guarantee.

In other words, not using the approach does not imply necessarily a bad development. For example, if a group is not using the approach, but the developers are constantly checking the accessibility elements, the resulting application may present good accessibility. Additionally, the Figure 7 displays the corresponding box-plot graph for the data distribution of the obtained results.

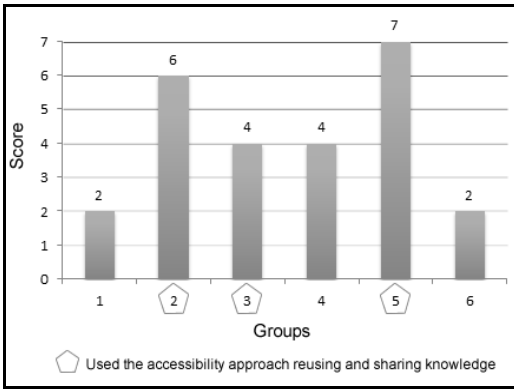

Figure 6. Score by group

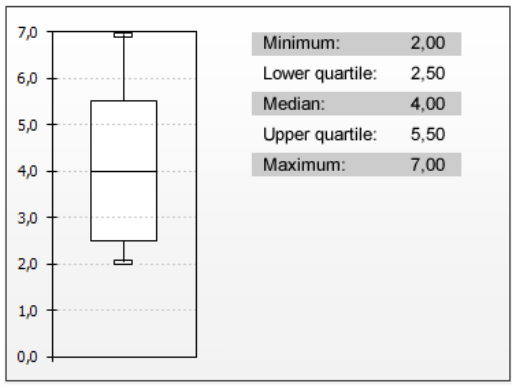

Figure 7. Data distribution

\begin{tabular}{|c|c|c|}
\hline Group & A & B \\
\hline 1 & 21 & No \\
\hline 2 & 11 & Yes \\
\hline 3 & 10 & Yes \\
\hline 4 & 18 & No \\
\hline 5 & 15 & Yes \\
\hline 6 & 13 & No \\
\hline
\end{tabular}

Table 3. DR

As observed in Figure 7, none of the groups obtained a zero score, indicating the importance of the initial training, regardless the approach used. Since this study was performed using DR as support, we presented: A) The number of arguments made in the DR for each group, and B) Whether the group uses the approach:

According to Table 3, there is no direct relationship between the quantity of captured records and the quality of the final product. However, the records are interesting for future projects, assisting in team communication and system maintenance. The fact of using or not the approach was not directly related to the 
amount of DRs recorded, since the group 1, without using the approach, obtained a low final score in accessibility checking but had the highest number of registered DRs (21).

In sum, DRs involved discussions about: technology being used, file structure, development environment, issues for the development of artifacts in HTML, interface design (colors, shapes, etc.) and search for ready components (calendar, captcha, etc.)

\subsection{Limitations and Threats}

Some limitations and threats were identified during the development of this research, as follows:

- Subjects Selection: as the selection was based on convenience sampling, we might have not selected the most representative set of subjects, to act as team members. This study took undergraduate students as participants, from the same university of the main author. They were considered to compose the project team. Therefore, not all subjects had previously been involved in industry projects, which could negatively have impacted on the study results.

- Project Scenario and Environment: the academic environment was chosen to perform such a project. It indeed hinders the generalization of results, since no industry practice was considered. Software engineering practice strives for data validation through not only toy, but instead real-world projects that offer real data and problems.

Thus, it is necessary to apply the practices from this academic project into a real project, in order to validate, refine and generalize the results.

\section{Conclusions}

In this study we carried out an experience of developing an application for which usable and accessible designs were expected. Several groups of developers performed the same task and we compared groups' behavior using and not using an approach developed since 2010 [2][3][4] with the support of previous knowledge, components and official guidelines.

There are many benefits for implementing accessibility in software applications, mainly in those available on the web, where the users can, sometimes, use the system without the help of experts.

This approach provided the developers the opportunity to learn and apply good accessibility practices. Such practices were collated in the development environment by recording design decisions related to the existing guidelines for the domain. As these practices may speed up development process, we organized them by topic and in order of importance to facilitate their reuse in other projects.

Only the groups using the approach suggested truly relevant contributions, as the calendar and the drop-down menu. This may be due to the short time available to perform the task, and to the fact that these groups took advantage of the available components repository.

A fact that is worth mentioning is that all groups faced difficulties in creating attractive layouts, probably because of their lack of knowledge on interface design. As 
observed, all groups were able to perform the proposed activities in this experiment. However, according to the evaluation criteria adopted, the goals were better accomplished by those groups that used the approach and, consequently, such groups presented more satisfactory results.

As future work we hope publish a full paper about the statistic test related to this experiment, which was omitted here due to the size limitation. In addition, we plan to integrate developing and knowledge managing tools in order to provide an adequate environment to good accessibility practices, as component use, live validation and scaffolding.

\section{References}

[1] Bigham, J. P.; Brudvik J. T. and Zhang B. 2010. Accessibility by demonstration: enabling end users to guide developers to web accessibility solutions. In Proceedings of the 12th ACM SIGACCESS Conference on Computers and accessibility (ASSETS '10). ACM, New York, USA, 35-42.

[2] Bittar, T. J., Amaral, L. A., and Fortes, R. P. M. AccessibilityUtil: a tool for sharing experiences about accessibility of web artifacts. 2011. In Proceedings of the 29th ACM international conference on Design of communication (SIGDOC '11). ACM, New York, NY, USA, 17-24.

[3] Bittar, T. J., Lobato, L. L., and Fortes, R. P. M. 2010. Guidelines2Model2Code: uma Abordagem para incorporação de diretrizes no desenvolvimento Web orientado a modelos. In: Actas da Conferencia IADIS Ibero Americana. WWW/INTERNET 2010, 2010, Lagoa - Algarve. Conferencia IADIS Ibero Americana. WWW/INTERNET 2010.

[4] Bittar, T. J., Neto, D., Lobato, L. L., Lucredio, D., and Fortes, R. P. M. 2010. Definição de metamodelos para Web incorporando boas práticas de acessibilidade e usabilidade. In: Proceedings of XXXVI Conferência Latino-Americana de Informática - CLEI 2010, Asunción.

[5] Chiuchi, C. A., Souza, R. C. G., Santos, A. B., and Valêncio, C. R. 2011. Efficiency and Portability: Guidelines to Develop Websites. In: Proceedings of the 23rd International Conference on Software Engineering \& Knowledge Engineering (SEKE), Miami - USA. 2011.

[6] Dutoit, A. H., McCall, R., Mistríkand, I. and Paech, B. 2006. Rationale Management in Software Engineering: Concepts and Techniques. A. H. Dutoit and R. McCall and I. Mistrík and B. Paech (eds.), Rationale Management in Software Engineering, Springer, 1-48.

[7] Falessi, D., Cantone, G. and Becker, M. 2006. Documenting design decision rationale to improve individual and team design decision making: an experimental evaluation. In Proceedings of the 2006 ACM/IEEE international symposium on Empirical software engineering (ISESE '06). ACM, New York, NY, USA, 134-143.

[8] Freire, A. P., Russo, C. M. and Fortes, R. P. M. 2008. A survey on the accessibility awareness of people involved in web development projects in Brazil. In 
Proceedings of the 2008 International Cross-disciplinary Conference on Web accessibility (W4A '08). ACM, New York, NY, USA, 87-96.

[9] Grubber, T. R. and Russel D. M.1991. Design Knowledge and Design Rationale: A Framework for Representation, Capture, and Use. Technical Report KSL 90-45, Knowledge Systems Laboratory, Stanford, California, 40p.

[10]Lazar, J., Feng J. H. andHochheiser H. 2010. Research Methods in HumanComputer Interaction. Wiley. ISBN 978-0-470-72337-1. 448 pages.

[11]Lee, J. 1997. Design Rationale Systems: Understanding the Issues. IEEE expert/Intelligent Systems and Their Applications, 12, 3, 78-85.

[12] Maclean, A., Young, R M., and Moran, T. 1989. Design rationale: the argument behind the artifact. SIGCHI Bull. 20, 247-252.

[13] Martín A., Cechich A. e Rossi G. 2011. Accessibility at early stages: insights from the designer perspective. In Proceedings of the International Cross-Disciplinary Conference on web Accessibility (W4A '11). ACM, New York, NY, USA, 9 pages.

[14] Paiva, D. M. B. and Fortes, R. P. M. 2005. Design Rationale in Software Engineering: A Case Study. In: Proceedings of the 17th International Conference on Software Engineering \& Knowledge Engineering, 2005, Taipei - Taiwan: United Daily News Digital Co. and Tung Tai University, 342-348.

[15] W3C. 1999. Web Content Accessibility Guidelines (WCAG) 1.0. Available in http://www.w3.org/TR/WCAG10/, accessed at January 2012.

[16] W3C. 2008. Web Content Accessibility Guidelines (WCAG) 2.0. Available in http://www.w3.org/TR/WCAG20/, accessed at January 2012.

[17] W3C. 2012. Techniques and Failures for Web Content Accessibility Guidelines 2.0. Available in http://www.w3.org/TR/WCAG-TECHS/, accessed at August January 2012.

[18] Wang, W. and Burge, J. E. 2010. Using Rationale to Support Pattern-Based Architectural Design. In Proceedings of the 2010 ICSE Workshop on Sharing and Reusing Architectural Knowledge (SHARK '10). ACM, New York, NY, USA, 1-8.

[19] Wohlin, C., Runeson, P., Höst, M., Ohlsson, M. C., Regnell, B., and Wesslén, A. Experimentation in software engineering: an introduction. Kluwer Academic Publishers, Norwell, MA, USA, 2000. 